\title{
Deep neural networks for human microRNA precursor detection
}

\author{
Xueming Zheng ${ }^{1}$, Xingli Fư ${ }^{2}$, Kaicheng Wang ${ }^{3}$ and Meng Wang ${ }^{4^{*}}$ (D)
}

\begin{abstract}
Background: MicroRNAs (miRNAs) play important roles in a variety of biological processes by regulating gene expression at the post-transcriptional level. So, the discovery of new miRNAs has become a popular task in biological research. Since the experimental identification of miRNAs is time-consuming, many computational tools have been developed to identify miRNA precursor (pre-miRNA). Most of these computation methods are based on traditional machine learning methods and their performance depends heavily on the selected features which are usually determined by domain experts. To develop easily implemented methods with better performance, we investigated different deep learning architectures for the pre-miRNAs identification.

Results: In this work, we applied convolution neural networks (CNN) and recurrent neural networks (RNN) to predict human pre-miRNAs. We combined the sequences with the predicted secondary structures of pre-miRNAs as input features of our models, avoiding the feature extraction and selection process by hand. The models were easily trained on the training dataset with low generalization error, and therefore had satisfactory performance on the test dataset. The prediction results on the same benchmark dataset showed that our models outperformed or were highly comparable to other state-of-the-art methods in this area. Furthermore, our CNN model trained on human dataset had high prediction accuracy on data from other species.

Conclusions: Deep neural networks (DNN) could be utilized for the human pre-miRNAs detection with high performance. Complex features of RNA sequences could be automatically extracted by CNN and RNN, which were used for the pre-miRNAs prediction. Through proper regularization, our deep learning models, although trained on comparatively small dataset, had strong generalization ability.
\end{abstract}

Keywords: miRNAs, DNN, Detection

\section{Background}

MiRNAs play import roles in gene expression and regulation and are considered to be important factors involved in many human diseases, e.g. cancer, vascular diseases or inflammation [1-3]. The biogenesis of miRNAs starts with the transcription of miRNA genes which forms primary miRNA hairpins (pri-miRNA). Then the pri-miRNAs were cleaved in the nucleus by RNase III enzyme Drosha, producing pre-miRNAs [4]. In an alternative pathway for miRNAs biogenesis, the pre-miRNA is from branched introns which are cleaved by debranching enzyme DBR1 $[5,6]$. After transportation to cytosol by Exportin-5, pre-miRNAs are further processed into

\footnotetext{
* Correspondence: mwang@just.edu.cn

${ }^{4}$ School of Computer Science, Jiangsu University of Science and Technology, Zhenjiang, China

Full list of author information is available at the end of the article
}

small RNAs duplexes by another RNase III enzyme Dicer $[7,8]$. Finally, the duplex loads into the silencing complex, wherein most cases one strand is preferentially retained (mature miRNA), while the other strand is degraded [9].

MiRNAs can be detected using experimental methods such as quantitative real-time PCR (qPCR), microarray and deep sequencing technologies [10-12]. All the experimental methods suffer from low specificity which needs extensive normalization. Furthermore, both qPCR and microarray can only detect known miRNAs since the primers for qPCR and the short sequences on microarray need to be predesigned [13].

Due to the difficulty of discovery of new miRNAs from a genome by existing experiment techniques, many ab initio computational methods have been developed [11]. Most of these classifiers which utilize machine learning 
algorithms such as support vector machines (SVM), are based on the carefully selected characteristics of premiRNAs [14-18]. The hand-crafted features of premiRNAs are the most important factors for the performance of the classifiers and therefore are generally developed by domain experts [19].

$\mathrm{CNN}$ and RNN, the two main types of DNN architectures, have shown great success in image recognition and natural language processing [20-22]. $\mathrm{CNN}$ is a kind of feedforward neural networks which contains both convolution and activation computations. It is one of the representative algorithms of deep learning, which can automatically learn features from raw input features [23]. The convolution layer, consisting of a combination of linear convolution operation and nonlinear activation function, is usually followed by a pooling layer which provides a typical down-sampling operation such as max pooling [24]. Through using multiple convolution and pooling layers, $\mathrm{CNN}$ models can learn patterns from low to high level in the training dataset [25].

Much as CNN is born for processing a grid of values such as image, RNN is specialized for processing sequential data [22]. One of the most popular RNN layers used in practical applications is called long short-term memory (LSTM) layer [26]. In a common LSTM unit, there are three gates (an input gate, an output gate and a forget gate) controlling the flow of information along the sequence. Thus, LSTM networks can identify patterns, which may be separated by large gaps, along a sequence [27].

Lots of CNN and RNN architectures have been developed to address biological problems and shown to be successful especially in biomedical imaging processing [28-31]. Here we designed, trained and evaluated the CNN and RNN models to identify human pre-miRNAs. The results showed that our proposed models outperformed or were highly comparable with other state-ofthe-art classification models and also had good generalization ability on the data from other species. Furthermore, the only information used in our models is the sequence combined with the secondary structure of pre-miRNAs. Our methods can learn automatically the patterns in the sequences avoiding the hand-crafted selection of features by domain experts, and therefore can be easily implemented and generalized to a wide range of similar problems. To the best of our knowledge, we are the first to apply $\mathrm{CNN}$ and RNN to identify human pre-miRNAs without the need for feature engineering.

\section{Results}

\section{Model's performance}

The CNN and RNN architectures for the pre-miRNAs prediction were proposed in this study. The detailed architectures and training methods of our deep learning models were shown in the methods section. For the training/evaluation/test splitting, the models were trained on the training dataset with enough epochs, evaluated on the evaluation dataset and finally the performance on the test dataset was shown as indicated in Table 1. In the 10-fold Cross Validation (CV), the performance was tested on each of the 10-folds, while the remaining 9-folds were used for training. For conciseness, we showed that the average performance along with standard error (SE) for the 10-fold CV experiments (Table 1).

As shown in Table 1, we got similar values of sensitivity (column 2), specificity (column 3), F1-score (column 4), Mathews Correlation Coefficients (MCC) (column 5) and accuracy (column 6) for these two kinds of dataset splitting strategies in each model. For both of the models, the values of sensitivity, specificity, F1-score and accuracy were mostly in the range of $80-90 \%$, while that of MCC in $70-80 \%$. In the $\mathrm{CNN}$ and RNN models, the prediction accuracy reached nearly 90\%. The RNN model showed better specificity, which exceeded $90 \%$, and poorer sensitivity (about 85\%).

For further comparisons, we plotted the ReceiverOperating Characteristic Curves (ROC) and the precision-recall curves (PRC) of different models for the training/evaluation/test splitting. All the parameters were trained on the training dataset and all the curves were drawn based on the test dataset. As shown in Fig. 1, the $\mathrm{CNN}$ model performed better reaching an area under the ROC curve (AUC) of 95.37\%, while the RNN model with an AUC of 94.45\%. The PRC also showed similar results.

Table 1 Performance of the proposed models

\begin{tabular}{llllll}
\hline Model (Data set partition) & Sen.(\%) & Spe.(\%) & F1(\%) & MCC(\%) & Acc.(\%) \\
\hline CNN (Training/Evalu./Test) & 88.83 & 88.28 & 88.83 & 77.11 & 88.56 \\
CNN (10-fold CV) & $89.58 \pm 4.72$ & $84.90 \pm 4.84$ & $87.53 \pm 1.38$ & $74.72 \pm 3.52$ & $87.24 \pm 1.80$ \\
RNN (Training/Evalu./Test) & 85.71 & 91.28 & 88.35 & 77.03 & 88.43 \\
RNN (10-fold CV) & $85.89 \pm 3.29$ & $91.14 \pm 2.75$ & $88.09 \pm 2.03$ & $77.04 \pm 3.66$ & $88.44 \pm 1.80$
\end{tabular}

Note: Classification performance of different models on the testing dataset was shown as sensitivity (column 2), specificity (column 3), F1-Score (column 4), MCC (column 5) and accuracy (column 6) respectively. For the 10-fold CV, the performance was shown as mean \pm standard error 

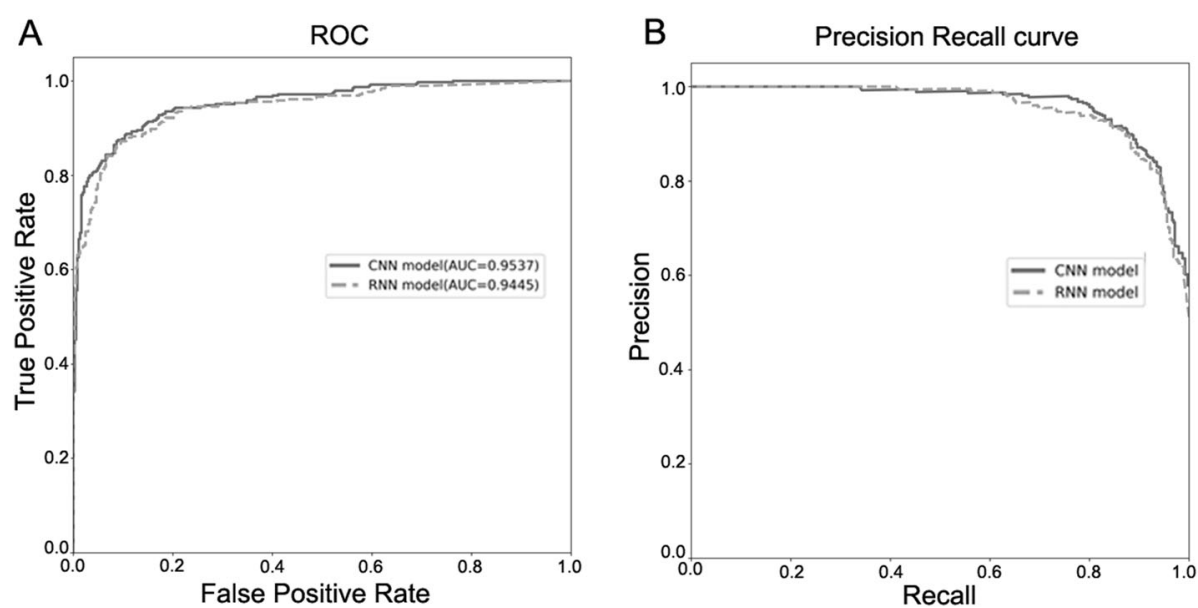

Fig. 1 ROC and PRC of proposed DNN models. ROC (a) and PRC (b) are shown as indicated. The AUC is also shown in (a)

\section{Performance comparison with other machine leaning methods}

For comparison, we referred to a newly published work done by Sacar Demirci et al. [19]. In their study, they assessed $13 \mathrm{ab}$ initio pre-miRNA detection approaches thoroughly and the average classification performance for decision trees (DT), SVM and naive Bayes (NB) was reported to be $0.82,0.82$ and 0.80 respectively. Following the same dataset splitting strategy, our models were retrained on stratified and randomly sampled training dataset (70\% of the merged dataset) and validated on the remaining $30 \%$ dataset. Here, we showed that the prediction results of some representative classifiers and our deep learning methods trained on the same positive and

Table 2 Comparison of model performance on the same benchmark datasets

\begin{tabular}{lll}
\hline Model & hsa & Pseudo \\
\hline This work $_{\text {CNN }}$ & 96 & 88 \\
This work $_{\text {RNN }}$ & 90 & 92 \\
Average $_{\text {DT }}$ & 97 & 93 \\
Consensus $_{\text {NB }}$ & 86 & 86 \\
Consensus $_{\text {DT }}$ & 99 & 90 \\
Ding $_{\text {NB }}$ & 88 & 84 \\
Average $_{\text {NB }}$ & 83 & 89 \\
NgDT $_{\text {Consensus }}$ & 89 & 89 \\
Batuwita $_{\text {NB }}$ & 97 & 96 \\
Bentwich $_{\text {NB }}$ & 86 & 83 \\
Ng $_{\text {NB }}$ & 92 & 71 \\
\hline
\end{tabular}

Note: Prediction results (percentage) of this work (rows 2-3) and the top ten models (rows 4-13) of the izMiR framework [19]. The values presented were true prediction rates (\%) (TPR) achieved for each model and dataset. Pseudo: negative data, from the coding region of human RefSeq genes, 8492 hairpins; hsa: positive data, Homo sapiens miRNAs, 1881 sequences negative datasets (Table 2). As shown in the table, our models had outperformed all the best individual methods (Ding $_{\mathrm{NB}}, \mathrm{Ng}_{\mathrm{DT}}$, Bentwich BB $_{\mathrm{NB}}$, Batuwita $\mathrm{NB}_{\mathrm{NB}}$ and $\mathrm{Ng}_{\mathrm{NB}}$ ), and yet were not as good as most of the ensemble methods (Average $\mathrm{DT}_{\mathrm{DT}}$, Consensus DT $_{\mathrm{T}}$ and Consensus).

\section{Classification performance on other species}

Since our models were trained and tested on human dataset, we wanted to know whether the trained classifiers could be applied to other species. We fed the welltrained CNN model with the pre-miRNAs sequences from Macaca mulatta, Mus musculus and Rattus norvegicus to perform classification. The pre-miRNAs of these species were downloaded from miRBase (http://www. mirbase.org/) and MirGeneDB [32] (http://mirgenedb. org/). For all these three species, more than $87 \%$ premiRNAs from miRBase were predicted to be true, while more $99 \%$ pre-miRNAs from MirGeneDB were correctly predicted (Table 3 ). The relatively higher prediction

Table 3 Prediction accuracy on pre-RNAs datasets from other species using the CNN model trained with human data

\begin{tabular}{lllll}
\hline Database & Species & $\begin{array}{l}\text { \# of pre- } \\
\text { miRNAs }\end{array}$ & $\begin{array}{l}\text { \# of correct } \\
\text { prediction }\end{array}$ & $\begin{array}{l}\text { Accuracy } \\
(\%)\end{array}$ \\
\hline $\begin{array}{l}\text { miRBase } \\
\text { release 22 }\end{array}$ & $\begin{array}{l}\text { Macaca } \\
\text { mulatta } \\
\text { Mus } \\
\text { musculus }\end{array}$ & 617 & 564 & 91.41 \\
& $\begin{array}{l}\text { Rattus } \\
\text { norvegicus }\end{array}$ & 495 & 436 & 87.60 \\
MirGeneDB & $\begin{array}{l}\text { Macaca } \\
\text { mulatta }\end{array}$ & 499 & 498 & 88.08 \\
& $\begin{array}{l}\text { Mus } \\
\text { musculus }\end{array}$ & 449 & 446 & 99.80 \\
& $\begin{array}{l}\text { Rattus } \\
\text { norvegicus }\end{array}$ & 414 & 411 & 99.33 \\
\hline
\end{tabular}


accuracy of Macaca mulatta might result from its closer evolutionary relationship with human.

The results showed that the proposed methods had good generalization ability on all the tested species. As we know, the quality of data is critical for deep learning. The high prediction accuracy might owe to the stricter standard for pre-miRNAs selection in MirGeneDB compared with those from miRBase.

\section{Discussion}

In this study, we showed that both $\mathrm{CNN}$ and RNN could automatically learn features from RNA sequences, which could be used for computational detection of human pre-miRNAs. Because of the small size of the dataset, the data quality and the vectorization method of input sequences would have great impact on the performance of the classifier. In the initial trial of this work, we only used the sequence of RNA to perform prediction. The results showed that although our DNN models could be successfully trained on the training dataset, there were high prediction error rates in the validation dataset, indicating low generalization ability. Although we tried different model structures and regularization methods, the big generalization error could not be reduced. This problem might result from the small sample size which couldn't be avoided. So, we combined the sequence and the secondary structure information as the input in our DNN models, which greatly minimized the generalization error. Good representations of data were essential for models' performance, although deep learning models could learn features automatically from data.

As we know, there are lots of hyperparameters for deep learning models, which needs to be determined before training. How to tune the hyperparameters to solve specific biological problems needs to be intensely studied in the future. So, we believe that great improvement could be made to identify pre-miRNAs in the future, although the models we proposed here performed very well.

\section{Conclusions}

In this work, we showed that both $\mathrm{CNN}$ and RNN can be applied to identify pre-miRNAs. Compared to other traditional machine learning methods, which heavily depend on the hand-crafted selection of features, $\mathrm{CNN}$ and RNN can extract features hierarchically from raw inputs automatically. In our deep learning models, we only used the sequence and the secondary structure of RNA sequences, which made it easy to implement. Furthermore, our models showed better performance than most SVM, NB and DT classifiers which were based on the handcrafted features. To investigate the performance on other species, we tested our CNN model with pre-miRNAs sequences from other species. The results showed that our methods had good generalization ability on all the tested species especially on the datasets from MirGengDB.

\section{Methods \\ Datasets preparation and partition}

The positive human pre-miRNA dataset (Additional file 1) containing 1881 sequences was retrieved from miRBase [33, 34]. The negative pseudo hairpins dataset (Additional file 2) was from the coding region of human RefSeq genes [35], which contained 8492 sequences. The secondary structures of the RNA sequences were predicted using RNAFolds software [36] and shown in the RNAFolds column of the datasets. Both the positive and the negative datasets were widely used for training other classifiers based mostly on SVM [19]. For the balance of datasets, we randomly selected the same number of negative sequences with that of positive ones. The selected negative and positive datasets were merged together and separated randomly into training (2408 sequences), validation (602 sequences) and test (752 sequences) datasets. In the10-fold CV experiments, the merged dataset was divided into 10 segments with about the same number of sequences (376 sequences). In each experiment, nine segments were used for training while the remaining one was used for evaluating the performance of the model.

\section{One-hot encoding and zero padding}

In the RNAFolds column of the supplementary datasets, the secondary structures were predicted by RNAfolds [33] and indicated by three symbols. The left bracket "(" means that the paired nucleotide/base at the $5^{\prime}$-end and can be paired with complimentary nucleotide/base at the 3 '-end, which is indicated by a right bracket")", and the "." means unpaired bases. In our deep neural networks, we only needed the sequences and the paring information. So, we merged the base ("A", "U", "G", "C") and the corresponding structure indicator ("(", “.", ")") into a dimer. Since there were four bases and three secondary structure indicators, we got twelve types of dimers. The newly generated features together with the labels were stored in the new files (Additional file 3 and Additional file 4). Next, we encoded the dimers with "one-hot" encoding (twelve dimension) and padding each sequence with the zero vector to the max length of all the sequences (180). So, each sequence could be represented by a vector with the shape of $180 \times 12 \times 1$, which was used in our supervised deep learning method (Fig. 2).

\section{Proposed deep neural network architecture The CNN architecture for the pre-miRNAs prediction}

The designed architecture of CNN was shown in Fig. 3a. In this model, the input sequences were first convolved by sixteen kernels with the size of four over a single 


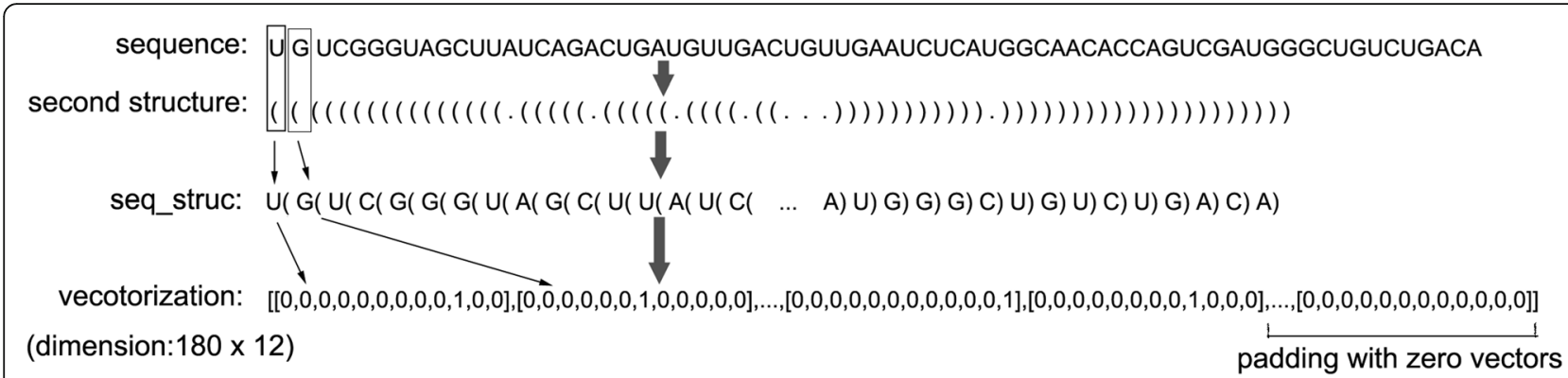

Fig. 2 One-hot encoding and vectorization of pre-miRNA sequence. The seq_struc is the combination of nucleotide/base and the corresponding secondary structure indicated with different symbols. The left bracket "("means paired base at 5'-end. The right bracket")" means paired base at 3'end. The dot "." means unpaired base. The encoded sequence is padded with zero vectors to the length of 180

spatial dimension (filters: 16, kernel size: 4), followed by the max pooling operation. Then the output tensors flowed through the second convolution layer (filters: 32, kernel size: 5) and max pooling layers, followed by the third convolution layer (filters: 64, kernel size: 6) and max pooling layers. All the max-pooling layers took the maximum value with the size of 2 . After convolution and max pooling layers, all the extracted features were concatenated and passed to a fully-connected layer with 0.5 dropout (randomly ignoring 50\% of inputs) for regularization in the training process. The dropout, a popular regularization method in deep learning, can improve the performance of our $\mathrm{CNN}$ model by reducing overfitting [37]. The last was the softmax layer whose output was the probability distribution over labels.

\section{The RNN architecture for the pre-miRNAs prediction}

In the recurrent neural networks (RNN) model, three LSTM layers with 128, 64 and 2 units respectively were used to remember or ignore old information passed along RNA sequences. Each LSTM unit is comprised of the following operations, where $\mathrm{W}$ and $\mathrm{U}$ are parameter matrices and $\mathrm{b}$ is a bias vector [27].

input gate: $\mathrm{i}_{\mathrm{t}}=\operatorname{sigmoid}\left(\mathrm{W}_{\mathrm{i}} \mathrm{x}_{\mathrm{t}}+\mathrm{U}_{\mathrm{i}} \mathrm{h}_{\mathrm{t}-1}+\mathrm{b}_{\mathrm{i}}\right)$.

forget gate: $\mathrm{f}_{\mathrm{t}}=$ sigmoid $\left(\mathrm{W}_{\mathrm{f}} \mathrm{x}_{\mathrm{t}}+\mathrm{U}_{\mathrm{f}} \mathrm{h}_{\mathrm{t}-1}+\mathrm{b}_{\mathrm{f}}\right)$.
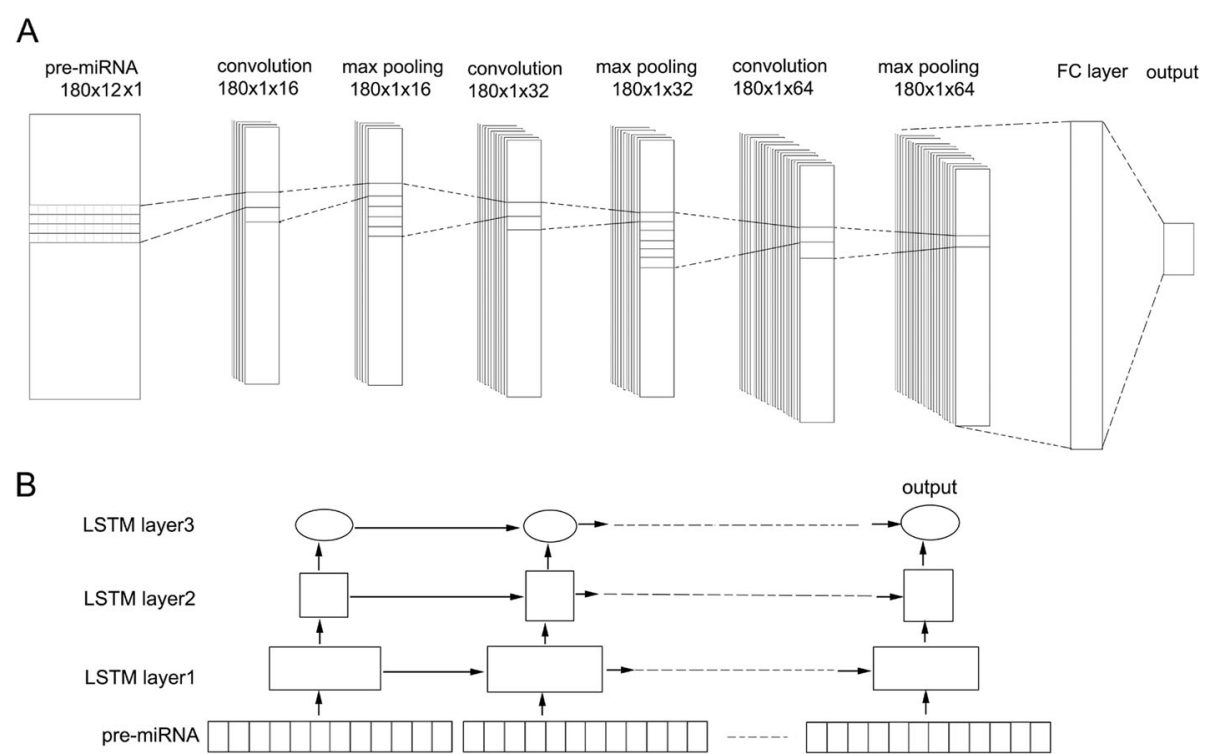

Fig. 3 The proposed CNN and RNN architectures for pre-miRNAs prediction. a. CNN model. The pre-miRNA sequence is treated as a $180 \times 12 \times 1$ vector. There are three cascades of convolution and max-pooling layers followed by two fully connected layers. The shapes of the tensors in the model are indicated by height $\times$ width $\times$ channels. FC: fully connected layer with 32 units. b. RNN model. Three LSTM layers with 128,64 and 2 units respectively are shown in the RNN. The final output is passed through a softmax function with the output of probability distribution over labels. In each time step along the pre-miRNA sequence, the LSTM cells remembered or ignored old information passed along the arrows. The output was the probability distribution over the true or false labels. 
transformation of input: $\mathrm{c}_{-} \mathrm{in}_{\mathrm{t}}=\tanh \left(\mathrm{W}_{\mathrm{c}} \mathrm{x}_{\mathrm{t}}+\mathrm{U}_{\mathrm{c}} \mathrm{h}_{\mathrm{t}-1}+\right.$ $b_{c}$ ).

state update: $c_{t}=i_{t} \cdot c_{-} i n_{t}+f_{t} \cdot c_{t-1}$.

$\mathrm{h}_{\mathrm{t}}=\mathrm{o}_{\mathrm{t}} \cdot \tanh \left(\mathrm{c}_{\mathrm{t}}\right)$.

output gate: $o_{t}=$ sigmoid $\left(W_{o} x_{t}+U_{o} h_{t-1}+V_{o} c_{t}+b_{o}\right)$.

For avoiding overfitting, the LSTM layers were regularized with randomly ignoring $20 \%$ of the inputs. The output tensors of the last LSTM layer were then passed through the softmax layer which gave the predicted probability over each label (Fig. 3b).

\section{Model training}

The loss function we used is the cross entropy between the predicted distribution over labels and the actual classification [38]. The formula is as follows.

$$
\text { Cross-entropy }=-\sum_{i=1}^{n} y_{i} \log s_{i}
$$

( $n$ : the number of labels, $y_{\mathrm{i}}$ : the actual probability for label i, $\mathrm{s}_{\mathrm{i}}$ : predicted probability for label i).

The aim of our machine learning was to minimize the mean loss by updating the parameters of the models. The models were fed by the training dataset and optimized by Adam algorithm [39]. The training processes were not stopped until the loss did not decrease any more. During the training process, the generalization error was also monitored using validation dataset. Finally, the learned parameters as well as the structures were stored.

\section{Methodology evaluation}

After training, we calculated the classifier performance on the test dataset in terms of sensitivity, specificity, F1Score, MCC and accuracy. (TP: true positive, TN: true negative, FP: false positive, FN: false negative).

Sensitivity:

$$
\text { Sen. }=\frac{\mathrm{TP}}{\mathrm{TP}+\mathrm{FN}}
$$

Specificity:

$$
\text { Spe. }=\frac{\mathrm{TN}}{\mathrm{TN}+\mathrm{FP}}
$$

F1-Score:

$$
\mathrm{F} 1=\frac{2 * \mathrm{TP}}{2 * \mathrm{TP}+\mathrm{FP}+\mathrm{FN}}
$$

MCC:

$$
\mathrm{MCC}=\frac{\mathrm{TP} * \mathrm{TN}-\mathrm{FP} * \mathrm{FN}}{\sqrt{(\mathrm{TP}+\mathrm{FN}) *(\mathrm{TN}+\mathrm{FP}) *(\mathrm{TN}+\mathrm{FN}) *(\mathrm{TP}+\mathrm{FP})}}
$$

Accuracy:

$$
\text { Acc. }=\frac{\mathrm{TP}+\mathrm{TN}}{\mathrm{TP}+\mathrm{TN}+\mathrm{FP}+\mathrm{FN}}
$$

Also, we plotted the ROC with the AUC and PRC for the training/evaluation/test splitting. With decreasing thresholds on the decision function used, corresponding false positive rates (FPR), TPR and precisions, recalls were computed. ROC curves were drawn based on a series of FPR and TPR, while PRC were based on precisions and recalls.

\section{Implementation and availability}

The implemented dnnMiRPre was well trained on the models using the training dataset and can be used to predict whether the input RNA sequence is a premiRNA. The dnnMiRPre's source code, which was written in Python with Keras library, is freely available through GitHub (https://github.com/zhengxueming/ dnnPreMiR).

\section{Supplementary information}

Supplementary information accompanies this paper at https://doi.org/10. 1186/s12859-020-3339-7.
Additional file 1. Human pre-miRNA.
Additional file 2. Pseudo hairpins.
Additional file 3. Human pre-miRNA with generated features.
Additional file 4. Pseudo hairpins with generated features

\section{Abbreviations}

AUC: Area under the ROC Curve; CNN: Convolutional Neural Networks; CV: Cross Validation; DNN: Deep Neural Networks; DT: Decision Trees; FN: False Negative; FP: False Positive; FPR: False Positive Rates; LSTM: Long Short-Term Memory; MCC: Matthews Correlation Coefficient; miRNAs: MicroRNAs; NB: Naive Bayes; PRC: Precision-Recall Curves; premiRNA: MiRNA precursor; pri-miRNA: Primary miRNA hairpins; qPCR: Quantitative real-time PCR; RNN: Recurrent Neural Networks; ROC: Receiver-Operating Characteristic Curves; SE: Standard Error; SVM: Support Vector Machines; TN: True Negative; TP: True Positive; TPR: True Positive Rates

\section{Acknowledgements}

We acknowledge anonymous reviewers for the valuable comments on the original manuscript. Lijun Quan at Soochow University has helped to proofread this manuscript.

\section{Authors' contributions}

$X Z$ and $M W$ designed and implemented the experiments. XZ wrote the manuscript. XF collected and preprocessed the data. KW wrote some of the source code. All the authors have approved the manuscript.

\section{Funding}

Clinical Medicine Science and Technology Development Foundation of Jiangsu University (JLY20180026).

The biomarkers selection and diagnosis of esophagus cancer based on data mining and hybrid models (KJS1739).

Scientific Research Foundation for the Startup Scholars in Jiangsu University of Science and Technology (Principal Investigator: Dr. Meng Wang).

Availability of data and materials

Models and datasets are made freely available through GitHub (https:// github.com/zhengxueming/dnnPreMiR). 


\section{Ethics approval and consent to participate}

Not applicable.

\section{Consent for publication}

Not applicable.

\section{Competing interests}

None of the authors has any competing interests.

\section{Author details}

'Department of Biochemistry and Molecular Biology, School of Medicine, Jiangsu University, Zhenjiang, China. ${ }^{2}$ Jiangsu University Health Science Center, Jiangsu University, Zhenjiang, China. ${ }^{3}$ Intensive Care Unit, HuiShan People's Hospital of Wuxi, Wuxi, Jiangsu Province, China. ${ }^{4}$ School of Computer Science, Jiangsu University of Science and Technology, Zhenjiang, China.

Received: 4 April 2019 Accepted: 3 January 2020

Published online: 13 January 2020

\section{References}

1. Mandujano-Tinoco EA, Garcia-Venzor A, Melendez-Zajgla J, Maldonado V. New emerging roles of microRNAs in breast cancer. Breast Cancer Res Treat. 2018;171(2):247-59.

2. Kir D, Schnettler E, Modi S, Ramakrishnan S. Regulation of angiogenesis by microRNAs in cardiovascular diseases. Angiogenesis. 2018:21(4):699-710.

3. Singh RP, Massachi I, Manickavel S, Singh S, Rao NP, Hasan S, et al. The role of miRNA in inflammation and autoimmunity. Autoimmun Rev. 2013;12(12):1160-5

4. Han J, Lee Y, Yeom KH, Nam JW, Heo I, Rhee JK, et al. Molecular basis for the recognition of primary microRNAs by the Drosha-DGCR8 complex. Cell. 2006;125(5):887-901

5. Ruby JG, Jan $\mathrm{CH}$, Bartel DP. Intronic microRNA precursors that bypass Drosha processing. Nature. 2007:448(7149):83-6.

6. Okamura K, Hagen JW, Duan H, Tyler DM, Lai EC. The mirtron pathway generates microRNA-class regulatory RNAs in drosophila. Cell. 2007; 130(1):89-100.

7. Lund E, Guttinger S, Calado A, Dahlberg JE, Kutay U. Nuclear export of microRNA precursors. Science. 2004;303(5654):95-8.

8. Park JE, Heo I, Tian Y, Simanshu DK, Chang H, Jee D, et al. Dicer recognizes the $5^{\prime}$ end of RNA for efficient and accurate processing. Nature. 2011; 475(7355):201-5

9. Rand TA, Petersen S, Du F, Wang X. Argonaute2 cleaves the anti-guide strand of siRNA during RISC activation. Cell. 2005:123(4):621-9.

10. Baker M. MicroRNA profiling: separating signal from noise. Nat Methods. 2010;7(9):687-92.

11. Tian T, Wang J, Zhou X. A review: microRNA detection methods. Org Biomol Chem. 2015:13(8):2226-38.

12. Dong $H$, Lei J, Ding L, Wen $Y$, Ju H, Zhang X. MicroRNA: function, detection, and bioanalysis. Chem Rev. 2013;113(8):6207-33.

13. Pritchard CC, Cheng HH, Tewari M. MicroRNA profiling: approaches and considerations. Nat Rev Genet. 2012;13(5):358-69.

14. Ng KL, Mishra SK. De novo SVM classification of precursor microRNAs from genomic pseudo hairpins using global and intrinsic folding measures. Bioinformatics. 2007:23(11):1321-30

15. Xue C, Li F, He T, Liu GP, Li Y, Zhang X. Classification of real and pseudo microRNA precursors using local structure-sequence features and support vector machine. BMC Bioinformatics. 2005;6:310.

16. Jiang $P$, Wu H, Wang W, Ma W, Sun X, Lu Z. MiPred: classification of real and pseudo microRNA precursors using random forest prediction model with combined features. Nucleic acids research. 2007;35(Web Server issue):W339-44

17. Rahman ME, Islam R, Islam S, Mondal SI, Amin MR. MiRANN: a reliable approach for improved classification of precursor microRNA using artificial neural network model. Genomics. 2012;99(4):189-94.

18. Batuwita R, Palade V. microPred: effective classification of pre-miRNAs for human miRNA gene prediction. Bioinformatics. 2009;25(8):989-95.

19. Sacar Demirci MD, Baumbach J, Allmer J. On the performance of premicroRNA detection algorithms. Nat Commun. 2017;8(1):330.

20. Krizhevsky A, Sutskever I, Hinton GE. ImageNet classification with deep convolutional neural networks. Commun ACM. 2017;60(6):84-90.
21. Albuquerque Vieira JP, Moura RS. In: Monteverde H, Santos R, editors. An Analysis of Convolutional Neural Networks for Sentence Classification; 2017

22. Mandic DP, Chambers JA. Recurrent neural networks for prediction learning algorithms, architectures, and stability. Chichester ; New York: Wiley; 2001. p. 285. xxi

23. Li LQ, Xu YH, Zhu J. Filter level pruning based on similar feature extraction for convolutional neural networks. IEICE Trans Inf Syst. 2018; E101D(4):1203-6.

24. Yu X, Yang J, Wang T, Huang T. Key point detection by max pooling for tracking. IEEE Transactions Cybernetics. 2015;45(3):444-52.

25. Zhang X, Zou J, He K, Sun J. Accelerating very deep convolutional networks for classification and detection. IEEE Trans Pattern Anal Mach Intell. 2016; 38(10):1943-55.

26. Gers FA, Schmidhuber E. LSTM recurrent networks learn simple contextfree and context-sensitive languages. IEEE Trans Neural Netw. 2001; 12(6):1333-40.

27. Hochreiter S, Schmidhuber J. Long short-term memory. Neural Comput. 1997;9(8):1735-80.

28. Tsiouris K, Pezoulas VC, Zervakis M, Konitsiotis S, Koutsouris DD, Fotiadis DI. A long short-term memory deep learning network for the prediction of epileptic seizures using EEG signals. Comput Biol Med. 2018;99:24-37.

29. Thireou T, Reczko M. Bidirectional long short-term memory networks for predicting the subcellular localization of eukaryotic proteins. IEEE/ACM Trans Comput Biol Bioinform. 2007:4(3):441-6.

30. Shen D, Wu G, Suk HI. Deep learning in medical image analysis. Annu Rev Biomed Eng. 2017:19:221-48.

31. Alipanahi B, Delong A, Weirauch MT, Frey BJ. Predicting the sequence specificities of DNA- and RNA-binding proteins by deep learning. Nat Biotechnol. 2015;33(8):831-8.

32. Chen W, Zhao W, Yang A, Xu A, Wang H, Cong M, et al. Integrated analysis of microRNA and gene expression profiles reveals a functional regulatory module associated with liver fibrosis. Gene. 2017;636:87-95.

33. Griffiths-Jones S, Saini HK, van Dongen S, Enright AJ. miRBase: tools for microRNA genomics. Nucleic Acids Res. 2008;36(Database issue):D154-8.

34. Kozomara A, Griffiths-Jones S. miRBase: annotating high confidence microRNAs using deep sequencing data. Nucleic Acids Res. 2014; 42(Database issue):D68-73.

35. Pruitt KD, Maglott DR. RefSeq and LocusLink: NCBI gene-centered resources. Nucleic Acids Res. 2001:29(1):137-40.

36. Hofacker IL. Vienna RNA secondary structure server. Nucleic Acids Res. 2003; 31(13):3429-31.

37. Baldi P, Sadowski P. The dropout learning algorithm. Artif Intell. 2014 210:78-122.

38. Wu X-H, Wang J-Q. Cross-entropy measures of multivalued neutrosophic sets and its application in selecting middle-level manager. Int J Uncertain Quantif. 2017:7(2):155-76.

39. Kingma D, Ba J. Adam: A Method for Stochastic Optimization. Computer Science; 2014

\section{Publisher's Note}

Springer Nature remains neutral with regard to jurisdictional claims in published maps and institutional affiliations.

Ready to submit your research? Choose BMC and benefit from:

- fast, convenient online submission

- thorough peer review by experienced researchers in your field

- rapid publication on acceptance

- support for research data, including large and complex data types

- gold Open Access which fosters wider collaboration and increased citations

- maximum visibility for your research: over $100 \mathrm{M}$ website views per year

At $\mathrm{BMC}$, research is always in progress.

Learn more biomedcentral.com/submission 